\title{
Zur weiteren Systematisierung der Fabrikplanung von Produktionskonzepten im turbulenten Umfeld
}

\author{
Claus-Gerold Grundig, Hermann Kühnle, Hans-Wolfgang Ahrend, Gerd Wagenhaus
}

\section{Inhaltsübersicht}

Steigende Marktdynamik und Globalisierung der Produktion erfordern eine ständige innovative Anpassung der Produktionskonzepte. Eine Vielzahl von Strategien der Gestaltung moderner Fabrik- und Produktionsstrukturen sind bekannt - die mögliche Umsetzungsbreite in der Industrie ist jedoch nur begrenzt ausgeschöpft. Als eine Ursache werden planungsmethodische Defizite angesehen, wodurch die Auswahl-, Entscheidungs- und Planungsabläufe verunsichert sind. Zudem zeigt sich, daß vor allem unter dem Paradigma zunehmender und sich immer schneller ändernder Ausgangsbedingungen und Planungsaktivitäten die neu geschaffenen Nutzenszeiträume der Produktionssysteme sich zunehmend verkürzen. Kaum akzeptabel jedoch sind im Kontext abnehmender Nutzensperioden zunehmende Aufwände für Fabrikplanungs- und Reorganisationsmaßnahmen. Hier gilt es neue Wege zu beschreiten, welche es ermöglichen hochspezialisiertes Fachwissen effizient einzusetzen und daruiber hinaus latent vorhandenes Erfahrungswissen, als Wissensbasis der Unternehmen, zu erschließen und in den Planungsprozeß einzubringen.

Ausgehend von einer kritischen Betrachtung des klassischen Ansatzes der Fabrikplanungssystematik wird ein inhaltlich- methodischer Neuansatz der vorhandenen Planungssystematik vorgestellt, der wesentliche Elemente innovativer Produktionskonzepte integriert und auch die Belange kleiner und mittelständischer Unternehmen (KMU) einschließt.
Der erforderliche Anpassungs- bzw. Veränderungsdruck - auch als Paradigmenwechsel [1] bezeichnet - begründet sich auf Ursachen wie:

- Globalisierung der Märkte und Standorte

- Kundendominanz (Käufermarkt)

- Dezentralisierung der Wertschöpfung

- Differenzierung der Kostenstrukturen

- Kurzzyklischer innovativer Wandel der Produkte / Ausrïstungen

Diese Entwicklungen, die sich teilweise überlagern und damit in der Regel potenzieren, wirken bei steigender Marktturbulenz direkt auf die Industrieunternehmen und die dort installierten Produktionskonzepte und müssen von diesen umgesetzt bzw. kompensiert werden.

Zur Bewältigung dieser Herausforderung wurden in der Fachwelt eine Vielzahl neuer, teils modifizierter Strategien der Fabrik- und Produktionssystemgestaltung entwickelt (vgl. Bild 1), die sämtlich auch Auswirkungen auf den Objektbereich der Fabrikplanung haben, d.h. zu modifizierten bzw. neuartigen Gestaltungs- und Organisationslösungen der Produktionskonzepte führen. Die Zielsetzungen innovativer marktflexibler Produktionskonzepte bestehen im Aufbau durchgängiger, dezentral strukturierter autonom agierender und kooperativ vernetzter flexibler Produktionssysteme bei einer ausgeprägten Markt- bzw. Kundenorientierung.

\section{Problemstellung}

Aus den Entwicklungen der Industriepraxis sowie der Fachliteratur ist bekannt, daß die Strategien der Gestaltung und Organisation von Fabrik- und Produktionssystemen beträchtlichen Wandlungen unterworfen sind.

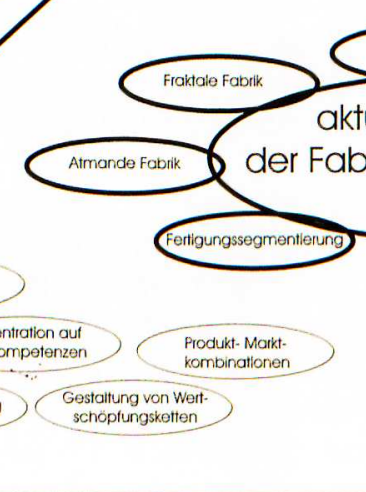

Nähere Betrachtungen dieser allgemein bekannten Strategien (Bild 1) zeigen teils neue, teils duale aber auch überlagernde Inhalte - allerdings bei in der Fachliteratur erkennbaren Defiziten hinsichtlich der Vorgabe von Einsatzkriterien, Planungskundenorientierung Abbau fentlgungstifef
systematiken und Zielsetzungen, was
für den Industrieanwender hinsicht-

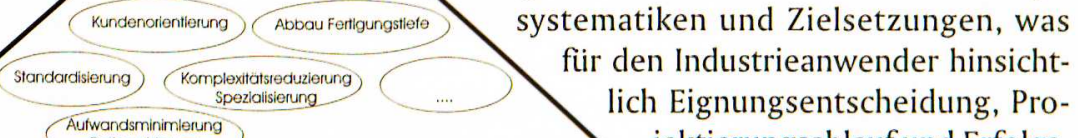
jektierungsablauf und Erfolgsabschätzung Probleme aufRationalisierung
Rering 9) wirft. Damit werden Fragestellungen aktuelle Strategien Rum EinfuihAmande Fabrik der Fabrik- und Produktions- rung rungs Manutacturno rand
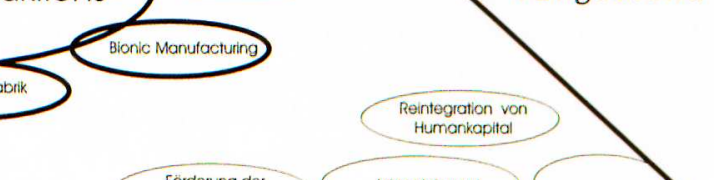
Förderung der
Innovationstanigkel 


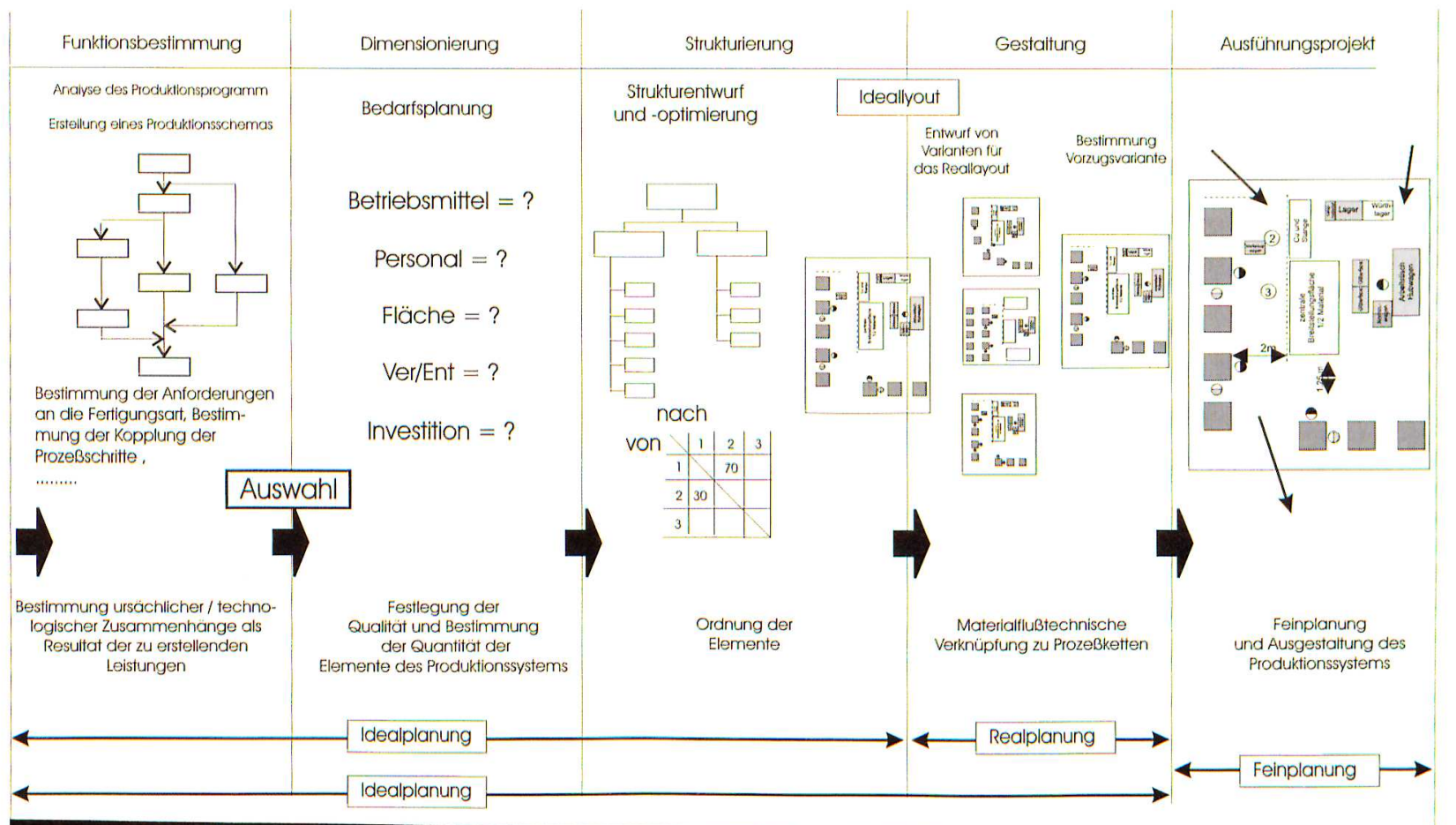

\section{Vom Groben zum Feinen}

Bild 2: Kernfunktionen der Fabrikplanungssystematik (nach [8])

und Wirkungserfolg aktueller innovativer Fertigungskonzepte relevant. Entsprechende Untersuchungen bzw. Projekte z.B. in [2] bis [7] bestätigen, daß bezuiglich der Einfuihrung und Umsetzung innovativer Produktionskonzepte teilweise beträchtliche Defizite bestehen. Auch wird deutlich, daß die Strategien zur Umsetzung neuer Produktionskonzepte sich teilweise als sehr unterschiedlich erweisen. Untersuchungen z. B. in [ 6 ] zeigen, daß nur ca. die Hälfte der Unternehmen innovative Produktionskonzepte zur Neugestaltung der Wertschöpfungsketten realisiert haben, wobei unter dem Aspekt der Erfolgsabschätzung auf Chancen und Risiken hingewiesen wird. Auf die verwirrende Vielzahl der Konzepte und den sich dabei ergebenden Orientierungsproblemen bis hin zu Mißerfolgen und Demotivation der Anwender wird in [ 7 ] ausfuihrlich hingewiesen.

Aus diesen Analysen ergibt sich, daß das Umsetzungspotential innovativer Produktionskonzepte in der Industrie nicht ausgeschöpft ist. Die Ursachen dieser Defizite sind unterschiedlich. Als eine potentielle Ursache werden in der Literatur [ 2 | bis [ 7 | aber auch von den Verfassern vor allem mangelnde methodische Vorgaben zum systematischen Entwurf, zur Eignungsüberprüfung und begleitenden Umsetzung der Produktionskonzepte angesehen. Dabei ist prinzipiell davon auszugehen, alle Strategien (Konzeptbegriffe) von Bild 1 wirken direkt auf Veränderungen im Objektbereich der Fabrikplanung woraus folgt - auch der Methodenbereich der Fabrikplanung ist zu hinterfragen hinsichtlich erforderlicher funktionell-inhaltlicher Neuansätze. Dabei gilt, neue innovative Gestaltungs- und Organisationsformen (Produktionskonzepte) erfordern folgerichtig eine veränderte methodische Herangehensweise an die Logik ihrer systematischen Entwicklung und Planung.

Von dieser Problemlage ausgehend werden nachfolgend
- basierend auf den in [8] dargestellten Grundprinzipien eines Neuansatzes der Fabrikplanungssystematik Weiterentwicklungen im Methodenbereich der Fabrikplanung fuir innovative Produktionskonzepte bei turbulentem Umfeld vorgestellt.

\section{Klassischer Ansatz der Fabrikplanungs- systematik}

Basierend auf systemtheoretischen Grundsätzen allgemeiner Problemlösungszyklen wurde der Fabrikplanungsablauf schon frühzeitig in allgemein anerkannte inhaltlich-methodisch abgrenzbare Planungsphasen gegliedert (vgl. z.B. [ 9 ] bis [ 16 ]). Ziele waren die Sicherung eines systematischen, schrittweisen Problemlösungs- und Projektbearbeitungsablaufes zur gezielten Lösungsfindung durch Vorgabe funktionell-logisch geordneter inhaltlich definierter Planungsphasen. Bei der Anwendung z.B. des 6-Phasen-Modells der Fabrikplanung (nach KETTNER [16]) erfolgt die Strukturierung der Planungsphasen in:

- Zielplanung

- Vorarbeiten (Analysen, Planungsdaten)

- Grobplanung (Planungsstufen Ideal-/ Realplanung) Erarbeitung Grobprojekte (Feasibility Studie) in Varianten

- Feinplanung Erarbeitung Feinprojekt (Vorzugsvariante)

- Ausfuihrungsplanung (Projektmanagement - Realisierungsablauf)

- Ausfuihrung (Projektrealisierung)

Systematik und Planungsinhalte der Grobplanung (Grobprojekt) können dabei verallgemeinernd - wie in Bild 2 dargestellt - zuruickgeführt werden auf die vier Kernfunktionen: 
- Funktionsbestimmung

- Dimensionierung

- Strukturierung

- Gestaltung,

die im Rahmen der funktionell-inhaltlich zu untersetzenden Planungsstufen Ideal- und Realplanung umzusetzen sind und die systematische Erarbeitung von Grobprojektlösungen gezielt unterstuitzen. Diese abstrahierenden Kernfunktionen des Fabrikplanungsablaufes ([9], [13], [14]), durch die wesentliche funktionell bedingte Planungsinhalte beschrieben werden, sind in Tafel 1 in ihrer Ablauflogik und Zuordnung zu den Planungsstufen Ideal- und Realplanung dargestellt.

Weiterhin erkennbar ist, daß die in der Literatur teilweise auftretenden unterschiedlichen Begriffe zur Bezeichnung von Funktionsinhalten sich bezüglich Inhalt und Einordnungslogik nahezu problemfrei den dargestellten Kernfunktionen zuordnen lassen, so daß der generalisierende Charakter der vier Kernfunktionen auch hier deutlich wird. Typisch für diesen Fabrikplanungsablauf ist, der Lösungsentwurf (feasibility Studie) für alle Planungsgrundfälle (Neubau, Umbau, Erweiterung, Rückbau) erfolgt im Rahmen einander bedingender, präzisierender Planungsphasen analytischer und synthetischer Planungsinhalte bei iterativen Durchläufen (Schleifenprozesse), wobei die Planungsergebnisse von Grob- zu Feininhalten entwickelt werden.

Wird diese historisch gewachsene grundlegende Planungssystematik aus heutiger Anwendersicht betrachtet, so ist erkennbar:

- die Planungssystematik ist neutral gegenüber der (den) jeweils vorliegenden Fertigungsart(-en)

- die Planungssystematik ist völlig lösungsunspezifisch bzw. objektunabhängig

- die Planungssystematik unterliegt einem rein funktions- bzw. bereichsbezogenem Denkansatz

- die Planungssystematik fokusiert auf direkte Produktionsfunktionen (Bearbeitungs- und Montage- prozesse) bei weitgehendem Ausschluß indirekter Produktionsfunktionen

- die Planungssystematik orientiert auf verrichtungsorientierte Fertigungsformen

- die Planungssystematik fokusiert auf zeit- und ortsstabile Geschäfts- und Produktionsstrukturen

Merkmale der nach dieser Planungssystematik verfolgten Lösungskonzepte sind z.B. Produktionsstrukturen, die rein unternehmensbezogen

- produktglobale indifferente Großbereiche (z.B. der Vorfertigung und Montage) hervorbringen, welche häufig gekennzeichnet sind durch eine

- hohe Arbeitsteiligkeit der Arbeitsabläufe (Taylorismus) in Verbindung mit der Orientierung auf

- zentrale Führungsstrukturen (Hierarchiestufen) und eine

- funktionell und räumliche Trennung direkter und indirekter Produktionsfunktionen sowie einer

- nachrangigen Gestaltung der Logistikprozesse.

Deutlich wird, dieser aus der Historie der Fabrikplanung herruihrende Planungsansatz, ist geprägt durch funktionsorientiertes Denken, tayloristische Prozeßstrukturierung, hierarchische Führungsstrukturen und Beschränkung auf „in house“-Funktionen des Unternehmens. Planungsziele waren der Aufbau ortsgebundener Produktionsunternehmen betont regional- bzw. unternehmenszentriert und begrenzter kooperativer Vernetzung (vgl. [17]). Darüiber hinaus lag meistens für die in der Vergangenheit durchzufuihrenden Planungsaktivitäten ein weitgehend konstantes und nur marginalen Schwankungen ausgesetztes Produkt- bzw. Teilespektrum vor. In Verbindung mit den in der Regel mittel- bis langfristigen Lebenszykluszeiten für die zu erstellenden Produktionssysteme ergab sich somit bisher kaum die Notwendigkeit Dynamik und Flexibilität in die Produktionssysteme mit „einzuplannen“.

Werden jedoch Erfordernisse und vorliegende Erfahrungen bei der Planung und Realisierung innovativer Pro-

\begin{tabular}{|c|c|c|c|}
\hline $\begin{array}{l}\text { Planungsstufen } \\
\text { (KETTNER [6]) } \\
\text { Planungsphase } \\
\text { Grobplanung }\end{array}$ & $\begin{array}{l}\text { Kernfunktionen } \\
\text { ([9] [13] [14]) }\end{array}$ & $\begin{array}{c}\text { Begriffszuordnung } \\
\text { (Literatur auszugsweise - [9] bis [16]) }\end{array}$ & Planungsinhalte \\
\hline \multirow[t]{3}{*}{ Idealplanung } & $\begin{array}{l}\text { Funktionsbestim- } \\
\text { mung }\end{array}$ & $\begin{array}{l}\text { Prinzipplanung } \\
\text { Arbeitsablaufschemaplanung } \\
\text { Verfahrensplanung } \\
\text { Prozeßbestimmung } \\
\text { Produktionsablaufschema } \\
\text { Funktionsschema }\end{array}$ & $\begin{array}{l}\text { Ermittlung der zur Produkterstellung erforderlichen } \\
\text { Funktionen und deren qualitative materialflußseitige } \\
\text { Verknüpfung (idealisiertes Funktionsschema) }\end{array}$ \\
\hline & Dimensionierung & Bedarfsplanung & $\begin{array}{l}\text { Bestimmung Typ und Anzahl der zur Funktionser- } \\
\text { füllung erforderlichen Elemente des Planungsob- } \\
\text { jektes }\end{array}$ \\
\hline & Strukturierung & $\begin{array}{l}\text { Systemplanung } \\
\text { Materialflußplanung } \\
\text { Anordnungsplanung } \\
\text { Konzeptplanung } \\
\text { Organisationstypen }\end{array}$ & $\begin{array}{l}\text { Festlegung von funktionsgerechten technisch- } \\
\text { organisatorisch und topologisch günstigen Struktur- } \\
\text { formen (Kopplung/Anordnung) von Ausrüstungen } \\
\text { (Bearbeitungs-, Montageprozesse) }\end{array}$ \\
\hline Realplanung & Gestaltung & $\begin{array}{l}\text { Integrationsplanung } \\
\text { Feinplanung } \\
\text { Layoutplanung } \\
\text { Bereichsplanung } \\
\text { Systemintegration }\end{array}$ & $\begin{array}{l}\text { Einordnung und Anpassung der Strukturformen in } \\
\text { das reale räumlich-funktionelle Umfeld, Ableitung } \\
\text { von Varianten (Layoutstrukturen), Auswahl Vor- } \\
\text { zugsvariante }\end{array}$ \\
\hline
\end{tabular}

Tafel 1: Kernfunktionen, Begriffszuordnungen und Planungsinhalte klassischer Fabrikplanungssystematik (Planungsphase Grobplanung) [8] 
jekte, wie z.B. der Planung fraktaler und segmentierter Strukturen bzw. integrierter Fertigungskomplexe (Projektbeispiele z.B. in [18] bis [22]) den dargestellten klassischen Planungsabläufen gegenuibergestellt, so zeigen sich beträchtliche Abweichungen bzw. „Sonderabläufe" im Planungs- und Entscheidungsablauf.

\section{Neuansatz der Fabrikplanungssystematik}

Der Neuansatz einer generalisierenden Fabrikplanungssystematik nimmt die Kriterien aktueller Kernstrategien der Strukturierung von Fabrik- und Produktionssystemen auf um inhaltlich-methodisch den gezielten Entwurf innovativer marktflexibler Produktionskonzepte zu sichern.

Als Kernprinzipien innovativer Produktionskonzepte sind anzusehen (vgl. z.B. [6], [10], [23], [28], [29], [30]) :

- Ganzheitlichkeit, Durchgängigkeit, Prozeßkettenbildung

- Kundenorientierung, Anpassungsfähigkeit,

- Innovationsfähigkeit und Reaktionsfähigkeit auf veränderte Bedingungen des Außenraumes

- Komplexitätsabbau, Dezentralisierung, Transparenz

- Wertschöpfungsorientierung, Logistikoptimierung

- Integration des Humanpotentiales als zentraler Gestaltungsansatz

Diese Kernprinzipien, deren Umsetzung in modernen Produktionskonzepten durchzusetzen ist, können folgenden Aufgabenkomplexen inhaltlich zugeordnet werden:

- Zielbestimmung und Festlegung der unternehmensextern und -intern wirkenden Leistungsmerkmale für neu zu konzipierender Produktionskonzepte. Ganzheitliche Planung des Gesamtprozesse der Produkterstellung und Vermarktung (bzw. Recycling) unter Einbeziehung aller Wertschöpfungs- und Logistikprozesse

- Entwurf durchgängiger Produktionsstrukturen bei Komplexitätsabbau und Verantwortungsdelegation durch geschäftsfeldbezogene marktorientierte Produktgruppenbildung. Aufbau von Produktionsbereichen mit Zielsetzungen weitgehender Komplettfertigung bei Konzentration auf Kernkompetenzen in Umsetzung begründeter Fertigungstiefe und kundenbezogener Produktionsstrategien.

- Innovative Gestaltung der Wertschöpfungs- und Logistikelemente bei Minimierung von Logistikaufwand und Schnittstellenanzahl. Integration und Erschließung von Humanpotential sowie indirekter Funktionen in den Produktionsprozeß. Sicherung ausgewogener Dezentralisierung und Autonomisierung von Produktionseinheiten bei begründeter Automatisierung.

Werden diese Aufgabenkomplexe den Planungsinhalten der klassischen Fabrikplanungssystematik gegenuibergestellt (vgl. Abschnitt 3), werden deutliche inhaltliche und methodische Abweichungen insbesondere aber auch Aufgabenerweiterungen erkennbar. Erfahrungen der Verfasser im Rahmen der Bearbeitung von Industrieprojekten zeigen, daß der erforderliche Neuansatz der Fabrikplanungssystematik hinsichtlich Planungslogik
(Systematik) sowie Planungsinhalten in den nachfolgenden drei Funktionskomplexen (FK) darstellbar ist [8]. Dabei wird vom allgemeinen Planungsfall „Mehrproduktfabrik bei Einzel- bis Massenfertigung und Mehrstufigkeit der Produktion" ausgegangen.

\section{Funktionskomplex A (FK A) - Prozessketten- modellierung}

Der Funktionskomplex A stellt den produktbezogenen Entwurf durchgängiger Prozeßketten (Leistungsketten) in Form ganzheitlich vernetzter kooperativer Prozeßabläufe (Unternehmensverbünde - Lieferanten / Finalproduzent / Kunde) der Produkterstellung und -vermarktung dar.

Die hier zu erstellenden Prozeßkettenpläne ermöglichen Analysen und erste Entscheidungen zu Wertschöpfungsund Kostenstrukturen der Sollprozesse. Bearbeitungs-, Montage-, Prüf-, Transport-, Umschlag- und Lagerprozesse werden technologisch- organisatorisch strukturiert hinsichtlich :

- Prozeßkettenlänge ( Lieferanten, Produzenten, Kunden, Entsorgung)

- Parallelitätsgrad (Verkürzung, Ressourcenkonzentration)

- Integrationsgrad (Teil-, Komplettbearbeitung)

- Erweiterung (insourcing)

- Verkürzung (Outsourcing)

- Vertauschung (örtlich / zeitlich)

- Substitution (Verfahren / Logistikelemente)

\section{Funktionskomplex B (FK B) - Entwurf Produktions- struktur}

Bildung von Produktgruppen (vertikale Strukturierung) Funktionelle Inhalte :

- Analyse und Zusammenstellung von identischen / ähnlichen Produkten zu Produktgruppen (-familien)

Kriterien:

- konstruktiv-technologische Ähnlichkeiten (Gruppen-, Familienbildung)

- Ähnlichkeiten der Prozeßkettenpläne

- Kundenorientiert - marktbezogen

- Stuickzahlbereiche / Produktionsvolumen (Prüfung der kapazitiven Auslastung der Prozeßkettenelemente und Abprüfung von Prioritätsverlusten durch Mehrfachzuordnung - Abgleich von Kapazitätsreserve und Zugewinn an Flexibilität)

- Absatzstrukturentwicklungen (Trend)

- Produkt- und Produktionsmix (Auflegestrategien)

\section{Bildung von Prozeßgruppen (horizontale Strukturierung)} Funktionelle Inhalte:

- Zuordnung Ausrüstungen (Bearbeitungs- und Logistikprozesse) zu den Prozeßketten der Produktgruppen (qualitative Dimensionierung)

- Bestimmung von Ausrüstungsanzahlen innerhalb der Prozeßketten (quantitative Dimensionierung)

Kriterien:

- Stufenarmut (Gesamtprozeß)

- Autonomie

- Flexibilität der Strukturen (Marktflexibilität)

- Ausreißerminimierung

- Kapazitätsauslastung 
- Ganzheitsprozesse (Lieferant - Abnehmer)

- Fertigungstiefenoptimierung

- Komplettbearbeitung

- Bestimmung qualifikatorischer Anforderungen an Personal

- Bestimmung fertigungstechnischer Anforderungen an Betriebs- und Hilfsmittel

\section{Ableitung Produktionsstruktur}

Funktionelle Inhalte:

Die Ableitung der Produktionsstruktur erfolgt als stufen- bzw. bereichsbezogene Zuordnung wirtschaftlicher Strukturformen (Fertigungsprinzipien, Fertigungsformen) je Prozeßgruppe. Entsprechend den erzielten Merkmalen der Prozeßgruppen sind hier deutlich unterschiedliche Strukturformen zuordenbar. Mögliche Betrachtungsebenen und daraus folgende Strukturformen sind (Auswahl):

1. verfahrens- bzw. gegenstandsorientierte Strukturen

- Punktfertigung

- Linienfertigung (Reihen- bzw. Fließfertigung)

- vernetzte Fertigung (flächenförmig)

Untergliederung in:

- ungeordnete vernetzte Fertigung - auch bekannt als Werkstattfertigung

- richtungsorientierte vernetzte Fertigung - auch bekannt als gegenstandsspezialisierter Fertigungsabschnitt

- und einfach vernetzte Fertigung (auch bekannt als Neststruktur)

- Verteilfertigung (raumförmig)

Untergliederung in:

- Zentralverteilungen

- Linienverteilung

- Flächenverteilung

2. NC-Strukturformen

- Flexible Fertigungszelle

- Flexible Fertigungsinsel

- Flexible Fertigungsstraße

- Flexibles Fertigungssystem

- Flexible Fertigungsverbundsysteme

3. Integrierte Strukturformen

- Fertigungsinsel (FI)

- Integrierte Fertigungsabschnitte (IFA)

- Integrierte gegenstandsspezialisierte Fertigungsabschnitte (IGFA)

4. selbstorganisierende dynamische Einheiten

- Fraktale

- Holone

- Bionic Organisation

- Verbünde

- Netzwerke

\section{Funktionskornplex C (FK C) - Modulgestaltung}

Im Funktionskomplex $\mathrm{C}$ erfolgt die technisch- organisatorische Gestaltung der gebildeten Wertschöpfungsund Logistikmodule entlang der Prozeßkette innerhalb der Prozeßgruppen.
- Endbestimmung von Bearbeitungstechniken (Wertschöpfungsmodule)

- Endbestimmung von Logistiktechniken (Transport-/ Fördersysteme, Lagersysteme, Kommissioniertechniken, Transporthilfsmittel, Umschlagtechniken u.a.)

- Endbestimmung des benötigten Qualifikationsprofiles fuir das Modul

- Feingestaltung des Layoutes (Anordnung, Fundamentierung, Ver- und Entsorgung, Arbeitsplatzgestaltung, Gestaltung des modulinternen Materialflusses)

- Festlegung von Organisationsprinzipien für die Materialbereitstellung /Materialflußlogistik (z.B. Bestimmung der Lager- und Belieferungsstrategie, just in time, KANBAN, zentrale / dezentrale PPS-Techniken)

- Auswahl prozeßgeeigneter PPS-Soft- und -HardwareLösungen auf Basis prozeßorientierter Anforderungschrakteristiken

- Festlegung von Arbeits- und Organisationsformen (gruppen- oder tayloristisch orientiert)

- Integration indirekter Funktionen - Festlegung des Umfangs unterschiedlicher Tätigkeiten - Gestaltung von Arbeitsinhalten (z.B. Qualitätswesen, Arbeitsvorbereitung, Instandhaltung)

Inhalte und Wirkungszusammenhang der dargestellten FK machen deutlich - im Vordergrund des Fabrikplanungsablaufes steht ganzheitliches Prozeßkettendenken zur Erfassung aller erforderlichen Wertschöpfungs- und Logistikelemente von der Produkterstellung bis zur Produktentsorgung. Dabei wird zunächst produktorientiert vorgegangen, d.h. Gesamtprozesse sind produktbezogen durchgängig (unternehmensübergreifend [24]) zu betrachten. Gleiche bzw. ähnliche Produkte / Produktelemente werden zu Produktgruppen zusammengefaßt bei Beachtung erforderlicher Markt- bzw. Absatzstrategien.

Unterschiedliche Produktgruppen führen im Regelfall zu unterschiedlichen Prozeßgruppen, wobei hier qualitative / quantitative Dimensionierungsfestlegungen basierend auf Grobentscheidungen zu Bearbeitungstechniken und Logistiksystemen durchzuführen sind. Ziele sind Ausreißerminimierung (Strukturwechselhäufigkeit) bei Sicherung wirtschaftlicher Auslastungsgrade der Bearbeitungs- und Logistikelemente. Erkennbar werden hier z.B. Möglichkeiten zur Bildung von unterschiedlichen Produktionsstrukturen. Weiterhin sind hier Entscheidungen zur Fertigungstiefe sowie zum Umfang von Zulieferkomponenten [25] zu treffen, durch die wirtschaftliche Wertschöpfungs- und Logistikketten festgelegt werden.

Anschließend erfolgt die präzisierte Modulgestaltung aller Wertschöpfungs- und Logistikelemente durch Endfestlegungen zu Fertigungsformen und Ausrüstungstypen einschließlich der Layoutgestaltung.

Diese Grobuibersicht zu den Funktionsinhalten des Neuansatzes macht deutlich - im Unterschied zur klassischen Fabrikplanungssystematik besitzt die Abarbeitung der FK im vorgestellten Neuansatz simultan-iterativen Charakter bei wechselseitig verflochtenem Entscheidungsablauf zwischen den drei FK. Dieser weiterentwickelte Planungsablauf besitzt folglich nicht rein 
sequentiell-iterativen Charakter bei dem die Planungsphasen konsequent sequentiell iterativ zu durchlaufen sind, sondern ist durch einen wechselseitig dynamisch vernetzten Ablauf der Planungs- und Entscheidungsprozesse zwischen den FK gekennzeichnet (vgl. Bild 3), d.h. Entscheidungen in der Projektbearbeitung sind immer in wechselseitiger Abhängigkeit der FK zu treffen.

So sind Entscheidungen zur quantitativen Dimensionierung z.B. von Verfahrenskomplexen innerhalb von Prozeßgruppen in ihrer Wirkung auf Vorgaben aus der Prozeßkettenbildung (FK A) und Produktgruppenbildung (FK B) sowie einzusetzender Ausrüstungstechniken (FK C) zu treffen. Gegebenenfalls sind Vorgabenkorrekturen erforderlich bzw. Zielsetzungen z.B. zur Autonomisierung oder Segmentierung von Produktionsbereichen sind nicht erreichbar - Korrekturen werden erforderlich.

Werden der klassische Fabrikplanungsansatz und der vorgestellte Neuansatz vergleichend gegenübergestellt, so werden die in Tafel 2 aufgeführten Zuordnungen erkennbar.
Die erzielbaren Strukturierungsergebnisse können sehr unterschiedlich sein. In Abhängigkeit von den Möglichkeiten der Produkt- und Prozeßgruppenbildung können sowohl produktuniverselle als auch produktspezifische Produktionsstrukturen je Fertigungsstufe bis hin zu durchgängigen Produktionssegmenten über mehrere Fertigungsstufen hinweg erzielt werden. Ergebnisse der Strukturbildung definieren damit die Möglichkeiten der Zuordnung von Fertigungsformen.

$\mathrm{Zu}$ beachten ist hierbei prinzipiell, daß der stark propagierte Aufbau segmentierter Strukturen (vgl. z.B. [26] eng gebunden ist an eine hinreichende Heterogenität der Produktionssortimente (Technologie- und Aufwandsmix) einschließlich entsprechender Stïckzahlstrukturen (Kapazitätsprofile).

Nur so kann der (oftmals vernachlässigte) Zielkonflikt der Segmentierung [27] gedämpft bzw. durch Ausgleichsmaßnahmen gelöst werden. Die Gestaltungsmöglichkeiten wirtschaftlich betreibbarer segmentierter Strukturen sind daher in der Industriepraxis als begrenzt anzusehen.

\begin{tabular}{|l|l|}
\hline Klassische & Neuansatz \\
Fabrikplanungssystematik & Fabrikplanungssystematik \\
(Kernfunktionen) & (Funktionskomplexe) \\
\hline Funktionsbestimmung & Prozeßkettenmodellierung (FK A) \\
\hline Dimensionierung / Strukturierung & Grundsatzentwurf - Produktionsstruktur (FK B) \\
\hline Gestaltung & Modulgestaltung (FK C) \\
\hline
\end{tabular}

Tafel 2: Vergleichende Gegenüberstellung - Klassischer Ansatz und Neuansatz der Fabrikplanungssystematik

Eine inhaltlich vergleichende Gegenüberstellung der Kernfunktionen (vgl. Abschnitt 3) mit den Funktionskomplexen (FK) zur Verdeutlichung des methodischen Neuansatzes zeigt:

\section{Funktionsbestimmung $\rightarrow$ Prozeßkettenmodellierung (FK A)}

Bei der Funktionsbestimmung werden nicht ausschließlich nur unternehmensinterne Funktionen der Wertschöpfungsprozesse sondern alle Wertschöpfungs- und Logistikelemente der gesamten Prozeßkette durchgängig produktbezogen abgebildet und modelliert.

\section{Dimensionierung / Strukturierung $\rightarrow$ Grundsatzentwurf Produktionsstruktur (FK B)}

Die Dimensionierung im klassischen Fabrikplanungsablauf beinhaltet Typvorauswahl und die kapazitive Auslegung von Teilsystemen (Betriebsmittel, Personal, Flächen, etc.). Für den Grundsatzentwurf der Produktionsstruktur sind diese Inhalte gleichermaßen im Rahmen der Prozeßgruppenbildung zu bearbeiten, allerdings nachgelagert bzw. immer im Wechselverhältnis zur Produktpruppenbildung und Prozeßkettenmodelierung. Projekte zeigen [18], [20], [21], daß z.B. Zielsetzungen zum Aufbau segmentierter Strukturen nur bei simultanen Abgleichen zu den Produktgruppenstrukturen und Prozeßketten zu lösen sind.
Gestaltung $\rightarrow$ Modulgestaltung (FK C)

Ergebnisse klassischer Planungssystematik sind hier das materialflußoptimierte (statisch / dynamisch) ermittelte Anordnungslayout (Varianten, Vorzugsvariante) bei anschließender räumlich-funktioneller Feingestaltung (Ausfuihrungsprojekt).

Aufbauend auf den Entwürfen von FK B erfolgt in FK C die Feingestaltung der Produktionsmodule (Bereiche, Segmente, Teilsegmente, Stufen) durch Bestimmung ausfuihrungsreifer Layoutstrukturen. Das schließt u. a. ein - die Endbestimmung von Bearbeitungstechniken sowie die Endbestimmung und Dimensionierung von Logistikelementen. Insbesondere gilt es hier, netzartige, dynamische Zulieferer-Finalproduzent-AbnehmerBeziehungen im Sinne ganzheitlicher Wertschöpfungsund Logistikketten durch eine gezielte technisch- organisatorische und informationstechnisch vernetzte Gestaltung der Module zu sichern. Das beinhaltet auch Auswahl und Einsatz z.B. von Simulations-, PPS- und BDE- Techniken zur Durchsetzung wirtschaftlicher marktflexibler Produktionskonzepte. Damit wird eine weitere Industrieerfahrung planungsmethodisch integriert - die Planung neuer Produktionskonzepte erfordert parallel zum Projektierungsprozeß (Fabrikplanung) die simultane Betrachtung der nach Inbetriebnahme durchzusetzenden organisatorischen Abläufe (Fabrikbetrieb). Dabei gilt prinzipiell, die Gestaltung der Pro- 
duktionskonzepte insbesondere unter logistischen Zielsetzungen legt Organisationsprinzipien fest, durch die wesentliche Auswahlkriterien der einzusetzenden PPSSysteme definiert werden.

In Tafel 3 ist eine inhaltliche Kurzbeschreibung der Planungsinhalte der Funktionskomplexe dargestellt.

\begin{tabular}{|c|c|}
\hline Funktionskomplex & Planungsinhalte \\
\hline $\begin{array}{l}\text { FK A } \\
\text { Prozeßkettenmodellierung }\end{array}$ & $\begin{array}{l}\text { Ausgehend vom Produktionssortiment (Mehrprodukt- } \\
\text { fabrik) wird je Produkt/Produktelement die materielle } \\
\text { Prozeßkette der Produkterstellung vom Zulieferer bis } \\
\text { Abnehmer (Kunde) erfaßt und dargestellt. Dabei er- } \\
\text { folgt die Festlegung fabrikinterner und externer Logi- } \\
\text { stik- und Wertschöpfungsfunktionen. }\end{array}$ \\
\hline \multicolumn{2}{|l|}{$\begin{array}{l}\text { FK B } \\
\text { Grundsatzentwurf Produktionsstruktur }\end{array}$} \\
\hline $\begin{array}{ll}\text { Produktgruppenbildung } \\
\end{array}$ & $\begin{array}{l}\text { Bildung von Produktgruppen auf Basis von konstruk- } \\
\text { tiv- technologischen Ähnlichkeitsmerkmalen, Prozeß- } \\
\text { kettenanalogie sowie Markt- und Kundenbezug }\end{array}$ \\
\hline Prozeßgruppenbildung & $\begin{array}{l}\text { Bildung von Prozeßgruppen durch qualitative und } \\
\text { quantitative Dimensionierungsentscheide }\end{array}$ \\
\hline Ableitung Produktionsstruktur & $\begin{array}{l}\text { Stufen- / bereichsbezogene Zuordnung wirtschaftli- } \\
\text { cher Strukturformen }\end{array}$ \\
\hline $\begin{array}{l}\text { FK C } \\
\text { Modulgestaltung }\end{array}$ & $\begin{array}{l}\text { Feingestaltung der Wertschöpfungs- und Logistikmo- } \\
\text { dule entlang der Logistikkette (Schnittstellenbe- } \\
\text { stimmmung) } \\
\text { Erstellung eines Anforderungsprofiles an Personal, } \\
\text { Betriebsmittel } \\
\text { Festlegung der innerhalb des Modules zu verrichteten } \\
\text { Tätigkeiten - Festlegung der Arbeitsorganisationsfor- } \\
\text { men }\end{array}$ \\
\hline
\end{tabular}

Tafel 3: Planungsinhalte der Funktionsgruppen (FK)

Planungsmethodisch ist bei Anwendung des Planungsneuansatzes prinzipiell wie folgt vorzugehen : Generalisierender Planungsablauf ist die aufeinanderfolgende Bearbeitung der Funktionskomplexe A, B, C, allerdings immer bei Durchsetzung der dargestellten wechselseitig vernetzten Planungs- und Entscheidungszusammenhänge der Funktionskomplexe (vgl. Bild 3).

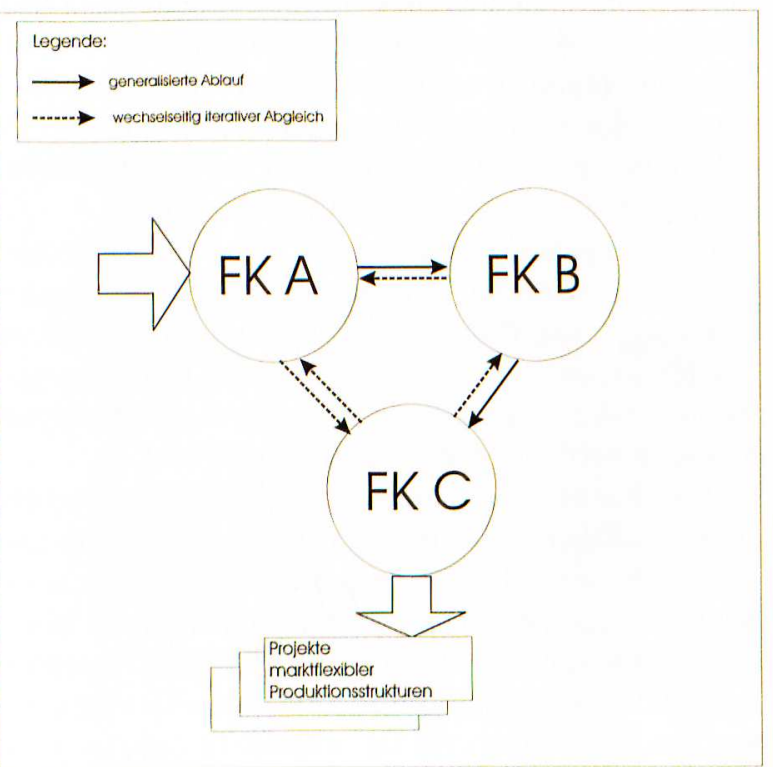

Bild 3: Abstimmung der Fabrikplanungsfunktionskomplexe - simultane Vernetzung

\section{Erfahrungen aus der betrieblichen Praxis}

Bei der betrieblichen Umsetzung innovativer Systeme des Fabrikbetriebes, wie z.B. der Produktion in selbstorganisierenden Struktureinheiten in Form von Fraktalen zeigt sich zunehmend, daß die Systematik der klassischen Fabrikplanung bereits in der Phase der Strukturfindung an die Grenzen des bisher machbaren stößt. Gerade die Dimensionierung und die darauf aufbauende Strukturierung der Produktion hängt hierbei im wesentlichen von den jeweils in den Unternehmen verfolgten Zielstellungen ab. Häufig ergibt sich gerade bei der Strukturfindung ein Widerspruch zwischen einer möglichst hohen kapazitiven Auslastung der Fertigung und der Schaffung von reaktionsschnellen und kundenspezifisch orientierter Organisationseinheiten.

Dieser Zielkonflikt verschärft sich, wenn aufgrund geringer Betriebsgröße und nur bedingt realisierbarerer Investitionen der Engpaßabbau durch Neudimensionierung bzw. durch Neuauswahl von Technologie/Technik nur begrenzt möglich ist. Der Fabrikplaner ist in solchen Fällen gezwungen einen Kompromiß zu finden, der eine weitgehende Kundenorientierung in allen Bereichen der Fertigung dennoch zuläßt. Genau hierbei spielt das interne Verständnis der Struktureinheiten zueinander ein entscheidende Rolle. So zeigte sich in einem Unternehmen des Maschinenbaues, daß es möglich und sinnvoll ist, kundenorientierte Montageeinheiten, die produktorientiert strukturiert und kundenorientiert ausgerichtet sind, mit einer verfahrenstechnisch strukturierten Vorfertigung, die als Dienstleistungszentrum im Sinne eines vorgeschaltenen Kunden im Unternehmen agiert, zu kombinieren. Die Zuordnung der Fertigungsformen zu den jeweiligen Struktureinheiten basierte hierbei auf kapazitiven Betrachtungen der Struktureinheiten (abgebildet als produktorientierte Prozeßketten) in Abhängigkeit vom Mobilitätsgrad der Arbeitsgegenstände bei Beachtung der durchgefuihrten Produktgruppenbildung (z.B. technologische/konstruktive Gleichheit der Erzeugnisse, ähnliche Fertigungsverfahren oder Arbeitsgänge bzw. ähnliche Montageabläufe). In der Praxis zeigte sich, daß einer durchgängigen produktorientierten Strukturierung der Fertigung, gerade bei KMU mit breiten Produktspektren, enge Grenzen gesetzt sind. Bild 4 stellt das prinzipielle Vorgehen zur Generierung unterschiedlicher Strukturierungsalternativen dar. 


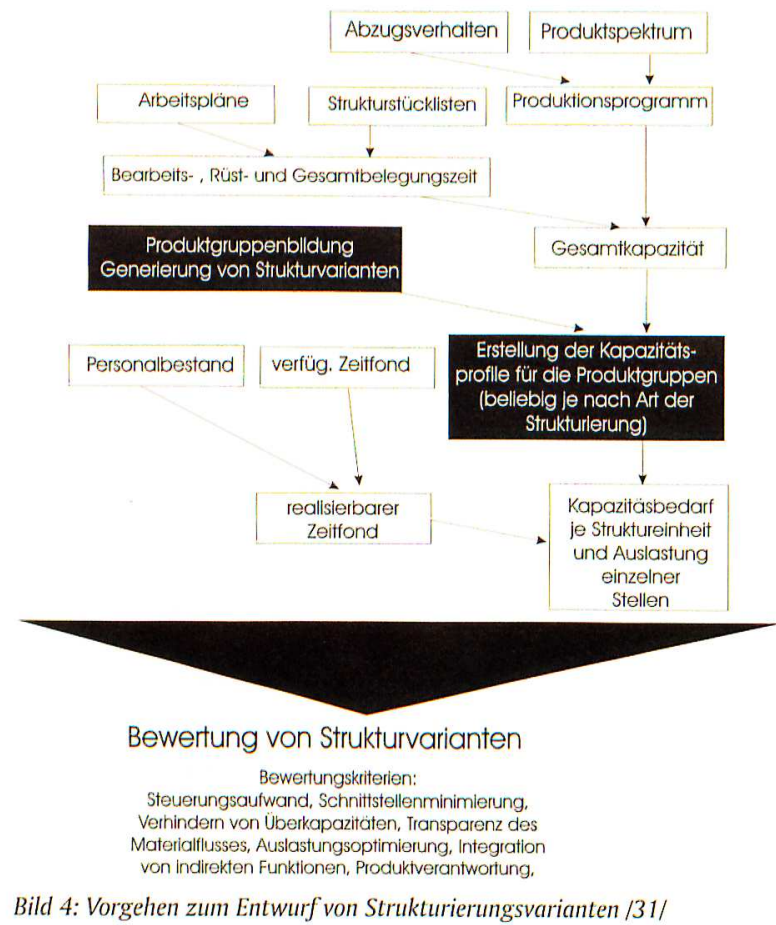

Ein Bewertungskriterium zur Strukturfindung ist die weitgehende redundanzfreie Zuordnung von Betriebsmitteln und Personal zu der zu schaffenden Struktureinheit. Eine Divergenz in der Zielstellung zwischen Element der Struktureinheit und dazugehörigem Zielsystem kann nicht akzeptiert werden. Ausdruck eines solchen Zustandes ist z.B. die nicht eindeutige und ausschließliche Zuordnung der Elemente zum übergeordneten System. Für die betriebliche Praxis ist damit relevant, daß eine "Splittung“ von „Engpaßmaschinen" in unterschiedliche Produktlinien, ohne eindeutige organisatorische sowie logistische Zuordnung zu einer definitiven Produktlinie auf Grund der weitgehenden Auslastung des Elementes durch diese Produktlinie, nicht durchzuführen ist. Nur unter der Prämisse der gezielten Schaffung von „Überkapazitäten" ist eine solcher Weg gangbar.

Die Ausrichtung und Ausgestaltung der einzelnen Struktureinheiten mit innovativen Arbeitsorganisationsmodellen (Ausfuihrungsprojekt) erfolgt dabei in der Regel unter aktiver Beteiligung der Mitarbeiter. Hierbei wird es einerseits ermöglicht Erfahrungswissen der gewerblichen Mitarbeiter in die neue Lösungsfindung einzubeziehen, andererseits werden über den Abgleich von Experten- und Erfahrungswissen suboptimale Systemlösungen weitgehend vermieden. Ein solches Vorgehen ermöglicht darüber hinaus eine Mobilisierung der Mitarbeiter für eine spätere aktive Mitarbeit bei der Organisation ihrer Arbeit. Erste Erfahrungen bei der Umgestaltung eines Montagesystems (Erstellung von Schaltschränken) bei Anwendung des vorgestellten Fabrikplanungsansatzes zeigten folgende Ergebnisinhalte in den Funktuionskomplexen:

\section{FK A}

- Analyse der technologischen Prozeßstruktur des Produktionsspektrums und der kapazitiven Belastung der Arbeitsplätze als Resultat des Produktionsprogrammes (z.B. prinzipieller technologischer Fluß des Auftragsdurchlaufes und Menge je Zeiteinheit ) - FK A - Prozeßkettenmodelierung
FK B

- Ermittlung der technisch/technologischen Notwendigkeiten und Zuordnungen von Betriebsmitteln und Tätigkeiten/Verrichtungen resultierend aus den konstruktiven Eigenschaften des Produkt bzw. bereits zusammengefaßten Produktspektrums - FK B - Produkt- und Prozeßgruppenbildung

\section{FK C}

- Ermittlung der ausrüstungsspezifischen Anforderungen an das neue Layout (z.B. schwere Pressen und benötigte Betriebsmittelflächen) - FK C - Modulgestaltung

- Erstellung einer räumlichen Grobstruktur der Betriebsmittel und / oder Arbeitsplätze und Erarbeitung der Raumstruktur der Arbeitsplätze (in Varianten, Eingliederung der Arbeitsplätze/Betriebsmittel [Organisationseinheiten] in die vorhandene Umgebung unter Beachtung von Mindestmaßen und Festlegung der Kernarbeitsplätze für die Montage, Zuordnung von Hauptarbeits- und Nebenarbeitsstellen) - FK C Modulgestaltung

- Erarbeitung einer maßstabsgerechten räumlichen Gestaltungslösung - Feingestaltung - FK C - Modulgestaltung

Häufig fuihren gerade in KMU, die erfahrungsgemäß oftmals unter dem hohen Druck operativer Tätigkeiten stehen, unzureichende Datengrundlagen zu erheblichen Aufwendungen für die materialflußoptimierte Gestaltung von Produktionssystemen. Um dennoch schnelle und möglichst zielsichere Lösungen zu erarbeiten, ist die Integration aller Mitarbeiter und deren aktive Teilnahme an der Systemgestaltung zwingend erforderlich. Durch ein systematisches Vorgehen nach dem dargestelltem Neuansatz und der Möglichkeit der aktiven Einflußnahme der Mitarbeiter auf die Layoutgestaltung können somit zügig innovative Lösungen entworfen und auf Eignung getestet werden.

\section{Zusammenfassung}

Die steigende Marktdynamik erzwingt eine ständige Neugestaltung bzw. Neuanpassung der industriellen Produktionskonzepte, insbesondere unter marktflexiblem Ansatz. Eine gezielte, innovative Über- oder Neuplanung der Produktionskonzepte setzt u.a. entsprechende methodische Neuansätze voraus - hier liegen Defizite vor.

Ausgehend vom klassischen Ansatz der Fabrikplanungssystematik wurde ein inhaltlich-methodischer Neuansatz vorgestellt. Dieser ist in drei Funktionskomplexe gliederbar, besitzt deutlich erweiterte Bearbeitungsinhalte und erfordert einen veränderten betont dynamisch vernetzten Bearbeitungsablauf.

Dieser Neuansatz wird gegenwärtig an unterschiedlichsten Projektbeispielen der Industriepraxis erprobt und inhaltlich-methodisch weiter präzisiert. Einige erste Anwendungserfahrungen wurden dargestellt.

Ziel ist es, dem in der Industrie tätigen Planungsingenieur eine zielfuihrende Planungssystematik zur Verfuigung zu stellen zur Unterstiitzung der Planungsund Projektierungsprozesse innovativer Fabrik- und Produktionskonzepte im turbulentem Umfeld. 


\section{Literatur}

[1] Warnecke, H.-J.: Revolution der Unternehmensstruktur, Springer-Verlag (1993)

[2] Neue Produktionskonzepte: Eine Diskussion macht noch keinen Sommer, VDI-Zeitschrift 139 (1997) 1/2, S. 21-23

[3] „Motion" - ein europäischer Veränderungsansatz, VDIZeitschrift 139 (1997) 5, S. 8-10

[4] Lay, G,; Dreher, C.; Kinkel, S.: Der Beitrag neuer Produktionskonzepte zur Sicherung der Wettbewerbsfähigkeit, ZWF 91 (1996) 12, S. 597-599

[5] Kohler, Oliver; Tschirsky, H.: Technologiemanagement interessant auch für kleine und mittelgroße Unternehmen, lo Management Zeitschrift 63 (1994) 11, S. 40-44

[6] Dreher, C.; Feig, J.; Harnischfeger, M.; Klimmer M.: Neue Produktionskonzepte in der deutschen Industrie, Physika-Verlag (1995)

[7] Lay, G.; Mies, C.: Erfolgreich reorganisieren (Unternehmenskonzepte aus der Praxis), Springer-Verlag (1997)

[8] Grundig, C.-G., Ahrend, H.-W.: Neuansatz der Fabrikplanungssystematik marktflexibler Produktionskonzepte, Werkstattstechnik 89 (1999) H. 6, S. 299-304

[9] Rockstroh, W.: Die technologische Betriebsprojektierung, Band 1, Verlag Technik Berlin (1977)

[10] Wirth, S.; Baumann, A.: Innovative Unternehmens- und Produktionsnetze, TU Chemnitz-Zwickau, Institut für Betriebswissenschaften und Fabriksysteme (IBF), Wissenschaftliche Schriftenreihe, Heft 8 (1996)

[11] Wiendahl, H.-P.: „Grundlagen der Fabrikplanung“, in: Betriebshütte (Produktion und Management) Teil 2, 7. Auflage, Springer-Verlag (1996)

[12] Aggeteleky, B.: Fabrikplanung, Hanser-Verlag (1990)

[13] Woithe, G.: „Betriebsgestaltung“, in: Betriebs- und Arbeitsgestaltung, Nutzensrechnung, Operationsforschung, Wissensspeicher für Technologen, Fachbuchverlag, Leipzig (1977)

[14] Schmigalla, H.: Fabrikplanung, REFA-Fachbuchreihe Betriebsorganisation, Carl-Hanser-Verlag (1995)

[15] Wiendahl, H.-P.: Analyse und Neuordnung der Fabrik, Springer Verlag (1991)

[16] Kettner, H.; Schmidt, J; Greim, H.-R.: Leitfaden der systematischen Fabrikplanung, Carl-Hanser-Verlag (1984)

[17] Spur, G.; Nackmayr, J.; Reising, W.: Optionen industrieller Produktionssysteme im Maschinenbau, in: Forschungsberichte der interdisziplinären Arbeitsgruppen der Berlin-Brandenburgischen Akademie der Wissenschaften (Band 4), Akademie-Verlag (1997)

[18] Küihnle, H.; Ahrend, H.-W.; Reising, W.: Fraktale Fabrik, Die dynamischen Veränderungen des Marktes von innen heraus bewältigen, Wt-Produktion und Management 87 (1997), S.1 11 1-1 14

[19] Eversheim, W., Kubin, G.; Munz, M.; Pelzer, W.; Schweitzer, G.: Ganzheitliche Unternehmensorganisation, VDIZeitschrift, 139 (1997) 7/8, S. 17-25

[20] Horn, V.; Trage, P.: Segmentierung steigert Leistung, ZWF 87 (1992) 6, S. 309-312

[21] Grundig, C.-G.: Das Lager in der Fertigung - Ein rechnergefuihrter, automatisierter Fertigungs-Komplex mit integriertem Lager- und Transport-System - Flexible Fertigung (1990), Heft 2, S. 66-69
[22] Wirtschaftliche Produktion, Praktische Umsetzung neuer Produktionskonzepte, AWF-Arbeitskreis (Herausgeber), Erich-Schmidt-Verlag (1995)

[23] Nedeß, Ch.; Mallon, J.; Strosina, Ch.: Die neue Fabrik, Handlungsleitfaden zur Gestaltung integrierter Produktionsstrukturen, Springer-Verlag (1995)

[24] Ahrend, H.-W.; Grundig, C.-G.: Tendenzen und Inhalte unternehmensuibergreifender Fabrikplanung, Wissenschaftliche Beiträge der Technischen Fachhochschule Wildau (1997), Heft 1, S. 40-47

[25] Ahrend, H.-W.: Ökologische Aspekte beachten bei der Reduzierung der Fertigungstiefe, Maschinenmarkt, 104 (1998) Nr.35, S. 34-47

[26] Wildemann, H.: Die modulare Fabrik, Kundennahe Produktion durch Fertigungssegmentierung, Verlag gfmt Gesellschaft AG (1 992)

[27] Hallwachs, U.: „Fertigungsinseln und -segmente als dezentrale Strukturkonzepte der Produktion", in: Handbuch Produktionsmanagement, Gabler-Verlag (1994)

[28] Neuendorf, H.; Scheffcyk,, H.; Vollmer, L.: Entwicklung einer wandlungsfähigen Fabrikstruktur, wt Werkstattstechnik 85 (1999) H1/21, S. 9-12

[29] Maßberg, W.: Sossna, F.: Gruppentechnologische Fertigungsstrukturen und wandlungsfähige Fabriken, Wt Werkstattstechnik 89 (1999) H1/2, S. 23-26

[30] Kühnle, H.: Simultane Fabrikgestaltung, Wt Werkstattstechnik 89 (1999) H 1/2, S. 13-17

[31] Kühnle, H,; Rietz, S.; Scholz, A.: STRUGTO - ein DVwerkzeug zur Produktionsstrukturbildung und -bewertung, Industrie Management 14 (1998)m 5, S. 53-57

\section{Autoren}

Prof. Dr.-Ing. Claus-Gerold Grundig

Technische Fachhochschule Wildau

Fachbereich Ingenieurwesen/Wirtschafts-

ingenieurwesen

Labor Fabrikplanung / Produktionsplanung und

-steuerung

Tel. (03375) 508171

Prof. Dr.- Ing. Hermann Kühnle

Institutsleiter des Fraunhofer-Institut für Fabrikbetrieb und -automatisierung und

geschäftsfuihrender Institutsleiter des Institutes für Arbeitswissenschaft, Fabrikautomatisierung und Fabrikbetrieb der Otto-von-Guericke-Universität Magdeburg Tel. (0391) 4090100

\section{Dozent Dr.-Ing. habil. Hans-Wolfgang Ahrend}

Otto-von-Guericke-Universität Magdeburg Institut fuir Arbeitswissenschaft, Fabrikautomatisierung und -betrieb (IAF)

Tel. (0391) 6712637

\section{Dipl.- Ing. Gerd Wagenhaus}

Abteilungsleiter Betriebsorganisation am FraunhoferInstitut für Faribkbetrieb und -automatisierung Tel. (0391) 6718615 